\title{
Correction to: Scalable and Communication- Efficient Decentralized Federated Edge Learning with Multi-blockchain Framework
}

\author{
Jiawen Kang, Zehui Xiong, Chunxiao Jiang, Yi Liu, Song Guo, \\ Yang Zhang, Dusit Niyato, Cyril Leung, and Chunyan Miao
}

\begin{abstract}
Correction to:
Chapter "Scalable and Communication-Efficient Decentralized Federated Edge Learning with Multi-blockchain Framework" in: Z. Zheng et al. (Eds.): Blockchain and Trustworthy Systems, CCIS 1267, https://doi.org/10.1007/978-981-15-9213-3_12
\end{abstract}

In the originally published version of the chapter 12 , the funding information in the acknowledgments section was incorrect. The funding information was removed. 